\title{
Real-life study showing better control of allergic rhinitis by immunotherapy than regular pharmacotherapy*
}

\author{
V. Droessaert', M. Timmermans', E. Dekimpe', S. Seys², J.L. Ceuppens ${ }^{2,3}$, \\ W.J. Fokkens ${ }^{4}$, P.W. Hellings s.,2, $^{1}$ \\ ' Clinical Department of Otorhinolaryngology-Head and Neck Surgery, KU Leuven, Belgium \\ ${ }^{2}$ Clinical Immunology, KU Leuven, Belgium \\ ${ }^{3}$ Clinical Department of Internal Medicine, Allergy Division, University Hospitals KU Leuven, Belgium \\ ${ }^{4}$ Department of Otorhinolaryngology, Academic Medical Center Amsterdam, the Netherlands
}

Rhinology 54: 214-220, 2016

DOI:10.4193/Rhino 14.282

*Received for publication:

November 23, 2014

Accepted: December 30, 2015

\begin{abstract}
Background: Treatment for allergic rhinitis (AR) aims at reducing the burden of allergic inflammation, either by suppression of the nasal inflammation with pharmacotherapy or by inducing tolerance via immunotherapy (IT). At present, we lack information on the comparison between the degree of symptom control in AR patients treated with IT and those on pharmacotherapy.
\end{abstract}

Aims: An observational study was conducted evaluating the degree of symptom control, the total and individual nasal symptom severity and current medication use at 3 years after starting either pharmacotherapy or subcutaneous immunotherapy (SCIT) for AR.

Methods: A total number of 800 patients diagnosed with AR between October 2007 and February 2010 at the Ear, Nose and Throat Unit and Allergology Clinical Department of the University Hospitals of KU Leuven, Belgium, were included. Among these patients, 120 had been started on IT at the time of their initial visit, and 680 were prescribed guideline-based pharmacotherapy. In 2013, patients were sent a questionnaire asking for the current severity of nasal symptoms using a visual analogue scale (VAS) score, duration of nasal symptoms and presence or absence of abnormal sleep, impairment of daily activities, sport, leisure, impaired functioning at work/school, troublesome symptoms, and current medication use. A VAS score for total nasal symptoms (TNS) was used to distinguish between controlled and uncontrolled AR.

Results: An overall response rate of 54\%. At 3 years after the initiation of the treatment, the IT group showed lower VAS scores for TNS than the pharmacotherapy group, with lower percentages of patients having a VAS score of equal or higher than 5 . The IT group consisted of more patients with mild AR than the pharmacotherapy group despite the higher percentage of polysensitization at the onset of treatment in the IT group. 18\% of the IT patients met the criteria of persistent AR whereas this was $51 \%$ amongst non-IT patients. Interestingly, $70 \%$ of IT patients did not use any medical treatment for AR anymore, whereas $61 \%$ of pharmacotherapy patients were still on medical treatment.

Conclusions: This observational study demonstrates that IT is associated with higher control of AR, reduced symptom severity and reduced medication use at 3 years after the onset of treatment. Therefore, this real-life study reinforces the clinical value of immunotherapy in allergic rhinitis.

Key words: allergy, rhinitis, treatment, control, uncontrolled rhinitis, allergic rhinitis 


\section{Introduction}

Pharmacotherapy for allergic rhinitis (AR) aims at reducing the burden of allergic inflammation, either by the suppression of the nasal and systemic symptoms associated with $A R$, or by the induction of tolerance to the allergens ${ }^{(1)}$. Different types of molecules like antihistamines, corticosteroids and leukotriene antagonists have anti-inflammatory properties and are recommended in international guidelines for the treatment of $A R^{(2)}$. The nasal or oral routes of administration are chosen by the clinician mainly on the base of expected benefit and clinical phenotype of AR. According to a survey of Cornelis et al. sublingual immunotherapy is the most frequently used form of IT in Belgium (3). The induction of tolerance is the goal of allergen-specific immuno-therapy (IT), being administered via the subcutaneous (SCIT), sublingual (SLIT) or oral route ${ }^{(3)}$. Tolerance to the inhalant allergens is obtained by the induction of regulatory $T$ lymphocytes and a deviation of the immune response away from the so-called T helper 2 cytokine profile ${ }^{(5)}$. Nasal provocation results in an increase in eosinophils in the nasal mucosa, but Muller et al. showed no change in the number of FoxP3 ${ }^{+} \mathrm{CD} 4^{+}$cells in a mono- or multiple-sensitized GP allergic individual, so the stronger clinical response to nasal provocation with GP is not related to differences in the number of regulatory $T$ cells in the nasal mucosa ${ }^{(6)}$. SCIT has been the standard IT modality since decades, with recent demonstration of efficacy of sublingual (4) and oral forms of IT ${ }^{(3)}$.

Pharmacotherapy as well as IT have both been proven to be effective on the level of symptom reduction as well as on inflammatory parameters like cytokine levels and histology scores in $A R^{(1)}$. The decision to prescribe pharmacotherapy or start with IT for AR is multi-factorial, involving the clinical phenotype of the patient, the sensitization profile of the patient, the degree of efficacy and/or tolerability of pharmacotherapy, as well as the overall perceived severity of the nasal and general symptoms. Prior to starting treatment, the expected benefits of pharmacotherapy and IT are ideally discussed with patients, as each treatment modality has its intrinsic advantages ${ }^{(5)}$. Until recently, we did not have good tools for the evaluation of symptom control in AR. Bousquet et al. were the first to demonstrate that a VAS score for TNS correlated well with the validated and widely used Rhinoconjunctivitis Quality of Life Questionniare ${ }^{(6)}$, hence offering the clinicians a convenient tool to use in clinical practice. In addition, Demoly et al. developed an allergic rhinitis control test (ARCT), which also is clinically applicable but more complex than the VAS for TNS $)^{(7)}$.

At present, we lack information on the comparison of the degree of symptom control between patients on pharmacotherapy and those on IT. Therefore, we conducted an observational study evaluating the degree of symptom control using the VAS for TNS in the patient population that had been started on either pharmacotherapy or IT 3 years prior to the study. Beside the
VAS for TNS, other clinically important parameters like severity and duration of symptoms and medication use were assessed.

\section{Materials and methods}

Study population

This explorative study was conducted between October 2012 and February 2013 at the Ear, Nose and Throat Unit and Allergology Clinical Department of the University Hospitals of KU Leuven, Belgium. The medical files from all patients that underwent skin prick test (SPT) between October 2007 and February 2010 were investigated.

Only patients who were really been treated for three years were included, so since the survey was conducted between October and February 2013, we used a variability of 4 months.

Inclusion criteria included all of the following items: having an age between 16 and 65 years old, being diagnosed with AR on the base of two symptoms suggestive of AR (sneezing, pruritus, nasal congestion and/or rhinorrea) and a positive SPT for at least one of the 18 most common inhalant allergens in Belgium (house dust mites, 7 different grass pollen, 3 kinds of tree pollen, animal dander (dog, cat, horse, rabbit), Alternaria, Penicillium and Cladosporium (HAL Allergy, Leiden, The Netherlands)). A positive SPT was defined by a wheal reaction of at least $3 \mathrm{~mm}$ in diameter or of a size equal to or larger than the positive control (histamine) after $15 \mathrm{~min}$, as recommended by Scadding, Hellings, Alobid, Bachert, Fokkens, van Wijk, et al. ${ }^{(8)}$.

All included patients had been diagnosed with moderate-tosevere and/or persistent AR, as defined by the ARIA guidelines (2), and rhinosinusitis and/or nasal anatomic pathology had been excluded by nasal endoscopy performed at the outpatient ENT clinic.

In all AR patients, pharmacotherapy was started as advocated in the ARIA guidelines ${ }^{(9)}$, unless they met the following indications for starting subcutaneous IT:

1. Severe symptoms of rhinitis in patients with high-dose allergen exposure.

2. Insufficient symptom control of AR despite the use of oral anti-histamines and intranasal corticosteroid sprays, and taking into account the possibility of allergen avoidance.

3. Bothersome adverse effects of medical treatment reported by the patients.

4. Patients preferring a long-term treatment that aims at a cure from allergic disease.

5. When animal allergen avoidance is impossible, for example for professional reasons.

Exclusion criteria for starting IT were asthma patients with persistent obstruction. The patients treated with pharmacotherapy will be named as the non-IT group later on in the text. The intensity of follow-up of both IT and non-IT patients had been dependent of the severity of remaining symptoms and the 
side effects of pharmacological or IT treatment. Non-IT patients were evaluated at 4 weeks after onset of treatment for evaluation of treatment response, and adaptation of treatment schedule. The follow-up was then performed by the referral medical doctor or by the ENT doctor or allergologist in case of persistent problems. Regarding IT, alum absorbed allergen solutions were obtained at HAL Allergy (Leiden) and ALK (Copenhagen). Allergens were mixed according to the sensitization pattern of the patient (with a maximum of three different allergen sources), and the allergen dose was progressively increased over a two year period (weekly injections for six months, two-weekly injections for 18 months), up to a maximum dose per allergen and per injection of $15.000 \mathrm{SQ}-\mathrm{U}$ (for ALK allergens) or $3000 \mathrm{AU}$ (for HAL pollen allergens) or $300 \mathrm{HU}$ (for HAL house dust mite extracts). The most important criteria for success were patient symptoms.

Hundred and twenty IT patients and 680 non-IT patients were sent a questionnaire. The non-responders were called after one month as a reminder and if necessary, a reminder questionnaire was sent to them.

Patients belonging to the non-IT group, reporting current or past IT, were excluded from the study, since the non-IT group consists of patients treated with pharmacotherapy only. During the treatment with IT, patients were allowed to use pharmacotherapy as add-on treatment depending on their symptoms. In the non-IT group, patients only used pharmacotherapy depending on their symptoms. We did not look for medication use during those 3 years, but only after 3 years, depending on their symptoms.

\section{Questionnaires}

The questionnaires were sent to all AR patients included in this study involving the following issues:

- A VAS score of 0-10 was used to measure each of TNS, i.e. nasal symptoms in general, we asked for any nasal complaint they suffered from, and individual nasal symptoms, i.e. nasal congestion, rhinorrhoea, sneezing, pruritus, headaches, reduction of sense of smell, cough, dyspnea and wheezing, experienced during the last 4 weeks. Visual analogue scales (VAS) have been used to rate the presence of symptoms or impairment of the daily activities (10). Bousquet et al. proposed this simple method for the quantitative evaluation of severity of AR, with a cut off value to distinguish between controlled $(<5)$ and uncontrolled $A R(\geq 5)^{(6)}$. - Presence or absence of abnormal sleep, impairment of daily activities, sport, leisure, impaired functioning at work/school and troublesome symptoms, to distinguish between mild i.e. none of the above symptom, or moderate/severe, i.e. at least one of the symptoms had to be present, AR.

- Duration of nasal symptoms, persistent, i.e. more than 4 days a week, and more than 4 consecutive weeks a year, intermittent i.e. less than 4 days a week and less than 4 consecutive weeks a
Table 1. Patients demographics in the IT group and pharmacotherapy (classified as the non-IT) group. With total number of patients, mean age and sensitization profile (mono- vs polysensitization) and different types of pharmacotherapy.

\begin{tabular}{|lcc|}
\hline & IT & Non-IT \\
\hline Number of patients (n) & 82 & 352 \\
\hline Men (\%) & $34(41.5)$ & $151(42.9)$ \\
\hline Women (\%) & $48(58.5)$ & $201(57.1)$ \\
\hline $\begin{array}{l}\text { Mean age (year, mean, standard } \\
\text { deviation) }\end{array}$ & $33.1(11.6)$ & $34.6(12.7)$ \\
\hline Atopy & $10(12)$ & $104(30)$ \\
\hline \multicolumn{1}{|c|}{ Mono-sensitized (\%) } & $72(88)$ & $248(70)$ \\
\hline \multicolumn{1}{|c|}{ Poly-sensitized (\%) } & $12(14)$ & $42(12)$ \\
\hline Asthma (\%) & $26(32)$ & $215(61)$ \\
\hline Pharmacological treatment (n) & $10(12)$ & $72(20)$ \\
\hline Anti-histaminicum (\%) & $4(5)$ & $57(16)$ \\
\hline Intranasal corticosteroids (ICS)(\%) & $12(15)$ & $87)$ \\
\hline Anti-histaminicum + ICS (\%) & & \\
\hline
\end{tabular}

Asthma was assessed by a Forced Expiratory Volume measuring. Atopy was diagnosed based on the presence of two symptoms suggestive of AR (sneezing, pruritus, nasal congestion and/or rhinorrea) and a positive skin prick test for at least one of the 18 most common inhalant allergens in Belgium (house dust mites, 7 different grass pollen, 3 kinds of tree pollen, animal dander (dog, cat, horse, rabbit), Alternaria, Penicillium and Cladosporium.

year.

- The current medication use at the time of evaluation, asking to specify the current treatment e.g. type of spray, or tablet, combination treatment or IT. Patients that had been started on IT were asked if the IT was still ongoing or finalized.

The Ethical Committee of the University Hospitals of KU Leuven approved the study protocol.

\section{Statistical analysis}

Descriptive statistics included frequencies and mean values. D'agostino and Pearson omnibus normality test was performed on data in both IT (median 1.9, interquartile range 0.7 - 3.4) and control group (median 3.2, interquartile range 1.5 - 6.1) and showed a nongaussian distribution ( $p$-value $=0.0001$ ). Comparison of outcome parameters between the IT and the non-IT group were performed using contingency analysis with Fisher's exact test. Comparison of VAS scores for different and total nasal symptoms were performed by Mann Whitney test. 

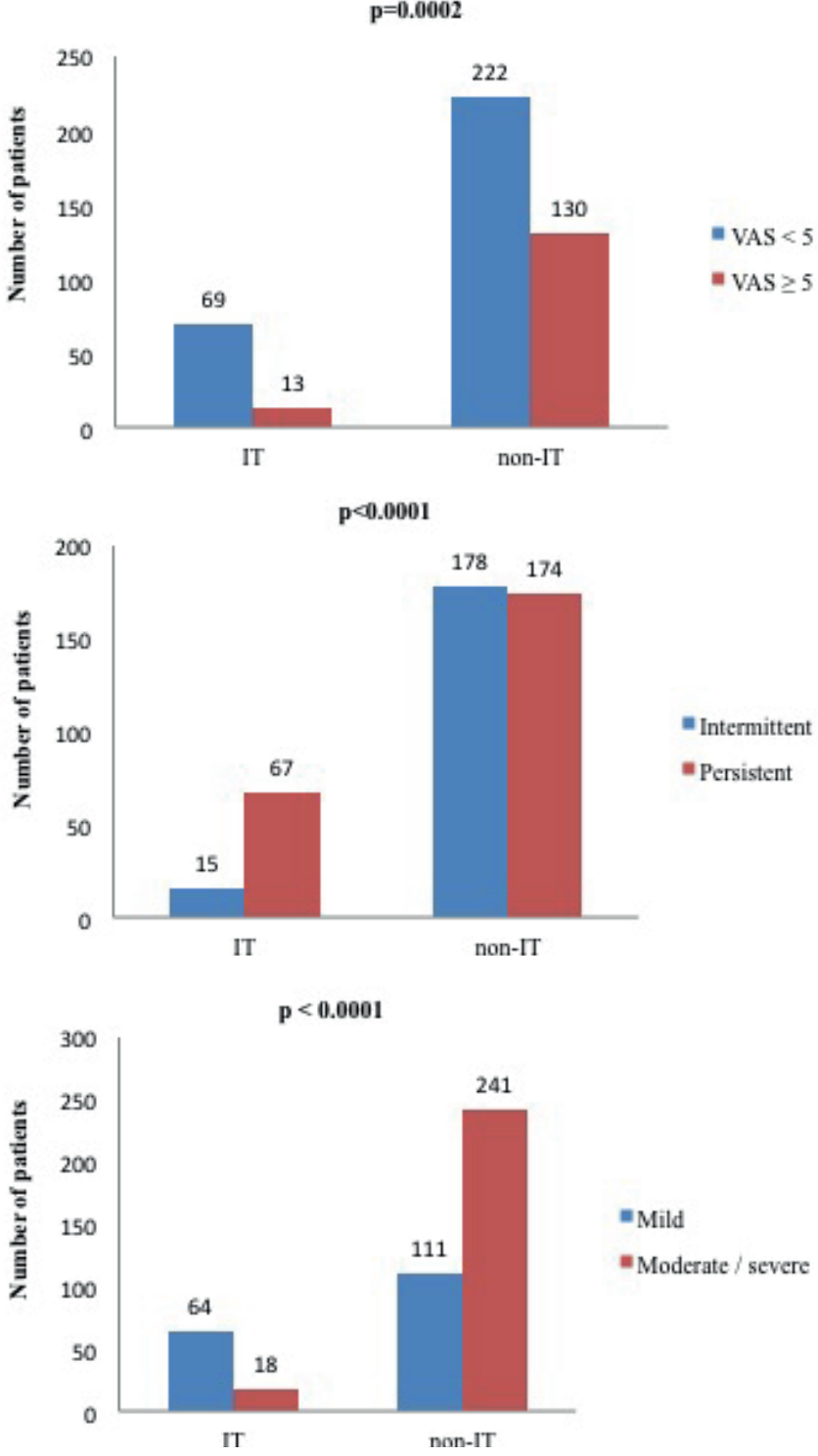

Figure 1. A) Severity of AR in relation to IT versus pharmacotherapy. Expression of severity by evaluation of the percentage of patients being controlled (VAS $<5$ ) vs uncontrolled (VAS $\geq 5$ ). B) Evaluation of duration of symptoms, i.e. intermittent vs persistent, of AR in IT and non-IT patients. C) Disease severity being mild vs moderate-severe in patients with pharmacotherapy and with IT treatment.

\section{Results}

\section{Study population}

A total response rate of $54 \%$ (434/800) was obtained, with $68 \%$ $(82 / 120)$ and $52 \%(352 / 680)$ of responders in the IT and non-IT group respectively.

The demographic characteristics of the patients are presented in Table 1. Demographic data were comparable between the two groups. Sensitization patterns differed between IT and non-IT patients, with poly-sensitization being more common in the IT group ( $88 \%$ (72/82) vs $70 \%$ (248/352), $p=0.001)$. Sensitization to HDM was more common in the IT group both for the mono-
Table 2. Total nasal symptoms and individual nasal symptoms (expressed as mean VAS score), i.e. nose congestion, rhinorrhea, sneezing, pruritus and associated symptoms.

\begin{tabular}{|lccc|} 
& \multicolumn{1}{c}{ IT } & non-IT & p-value* \\
\hline TNS $(\mathrm{cm})$ & $2.5 \pm 2.3$ & $3.7 \pm 2.8$ & $<0.0001$ \\
\hline Nose congestion $(\mathrm{cm})$ & $2.7 \pm 2.3$ & $3.3 \pm 1.2$ & $<0.0001$ \\
\hline Rhinorrhea $(\mathrm{cm})$ & $2.3 \pm 2.1$ & $3.6 \pm 1.2$ & $<0.0001$ \\
\hline Sneezing $(\mathrm{cm})$ & $2.6 \pm 2.2$ & $3.7 \pm 1.1$ & $<0.0001$ \\
\hline Pruritus $(\mathrm{cm})$ & $2.3 \pm 1.9$ & $4.0 \pm 1.1$ & $<0.0001$ \\
\hline Loss of smell $(\mathrm{cm})$ & $2.4 \pm 2.4$ & $3.8 \pm 1.1$ & $<0.0001$ \\
\hline $\begin{array}{l}\text { Lower airway } \\
\text { symptoms }(\mathrm{cm})\end{array}$ & $2.2 \pm 2.2$ & $3.9 \pm 1.2$ & $<0.0001$ \\
\hline
\end{tabular}

Legend: Visual Analogue Score (VAS) was used to assess nasal symptoms. TNS: total nasal symptoms. *Mann Whitney test was used to compare IT and non-IT group.

sensitized ( $33 \%$ vs $16 \%$ ) as well as for the poly-sensitized AR patients (74\% vs $54 \%)$.

\section{Degree of control of rhinitis at a mean interval of $\mathbf{3}$ years after start of therapy}

Control is defined as a disease state in which the patients do not have major symptoms anymore or the remaining symptoms are not regarded as bothersome, with VAS scores for TNS being a good tool ${ }^{(10)}$. In the IT group, $84 \%$ of patients had a VAS for TNS of $<5$, whereas this was lower in the non-IT group (63\%, Figure $1 \mathrm{~A})$. Alternatively, only $16 \%$ of IT patients noted a VAS score for TNS $\geq 5$ compared to $37 \%$ of non-IT patients. Of note, 2 of the 13 IT patients with a VAS for TNS of $>5$ suffered from asthma, with a VAS score $\geq 5 / 10$ for shortness of breath and wheezing during the last four months.At 3 years after starting treatment, mean VAS scores for TNS in the IT group $(n=82)$ were significantly lower compared with non-IT patients $(n=352)(2.5 \pm 2.3$ vs $3.7 \pm 2.8$, p-value < 0.0001).

The mean VAS scores for TNS, and the individual nasal symptoms for IT and non-IT group are presented in table 2. In the IT group, $84 \%$ of patients had a VAS for TNS of $<5$, whereas this was statistically lower in the non-IT group (63\%, Figure $1 \mathrm{~A})$ ( $p$-value $<0.0002$ ). Of note, 2 of the 13 IT patients with a VAS for TNS of $\geq 5$ suffered from asthma, with a VAS score $\geq 5 / 10$ for shortness of breath and wheezing during the last four months (Table 2).

Severity and duration of rhinitis at a mean interval of $\mathbf{3}$ years after start of therapy

In the IT group, 15 patients (18\%) versus 178 non-IT patients (51\%) were considered as having a persistent rhinitis (Figure 1B). Meaning that in the IT group statistically significant less patients 


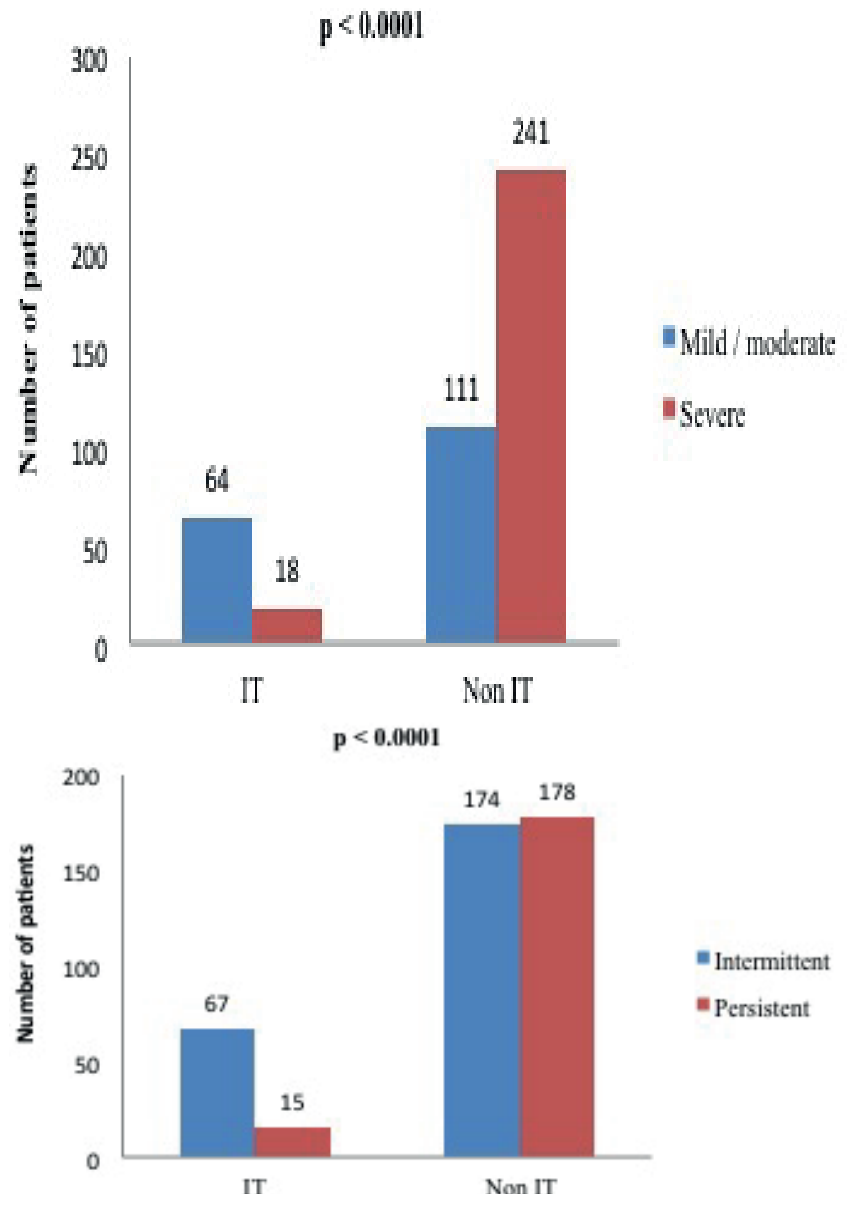

Figure 2. A, Severity, i.e. mild/moderate vs severe, of AR three years after treatment with IT versus non-IT. B. Duration of AR, i.e. intermittent vs persistent three years after treatment with IT versus non-IT.

suffered from persistent rhinitis ( $p$-value $<0.0001$ ).

The ARIA classification of AR is based on duration and severity of AR symptoms ${ }^{(2)}$. In the IT group, 15 patients (18\%) versus 178 non-IT patients (51\%) reported symptoms for $\geq 4$ days a week and $\geq 4$ consecutive weeks per year, and were categorized as having persistent rhinitis (Figure 1B).

Patients of the IT group with intermittent AR showed significantly lower mean VAS $(2.02 \pm 1.9)$ scores for total nasal symptoms compared to patients with persistent symptoms $(4.8 \pm 2.6)$ ( $p$ value $<0.0001$ ).

Significantly less patients in the IT group (22\%) met ARIA criteria for moderate-to-severe AR versus 241 patients (68\%) in the nonIT group (Figure $1 \mathrm{C}$, $\mathrm{p}$-value $<0.0001$ ).

Comparison between the IT and non-IT groups showed a significant difference in the ARIA based severity and duration classification of the AR at 3 years after start of therapy ( $p$-value $<0.0001)$.

After 3 years of treatment, significantly less IT-patients suffered from severe or persistent AR compared to the non-IT group (05, Figure $2 A-B, p$-value $<0.0001$, Fisher's exact test) $1 B$ and $C$ )).

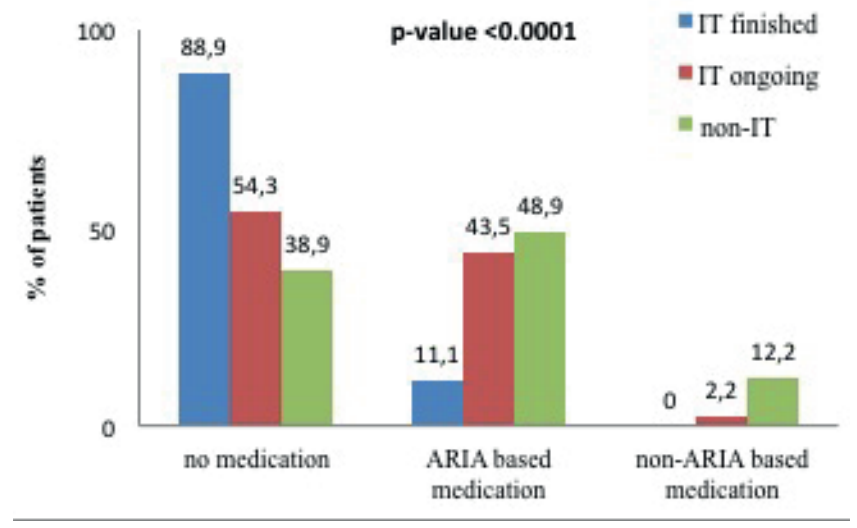

Figure 3. Percentage of patients using medication for AR in relation to treatment with IT (finished or going) and pharmacotherapy.

Current medication use at 3 years after start of therapy The IT group was subdivided in patients with ongoing IT ( $n=46)$ and finished IT $(n=36)$. The current medication use at 3 years after diagnosis of AR in those two groups was compared to the non-IT group (Figure 3). In the IT group, $29.3 \%$ of the patients used ARIA-based medication, 1 patient used a decongestion nasal spray and $69.5 \%$ were not on any medical treatment. Sixty-one percent of patients in the non-IT group were still on medical treatment. Eighty-eight point nine percent of the patients who finished the IT and $54.3 \%$ of those who were still on IT did not use any anti-allergic medication compared to $38.9 \%$ of the patients in the non-IT group. Only $11.1 \%$ of the IT-finished and $43.5 \%$ of the current IT-group used ARIA based medication compared to $48.9 \%$ in the non-IT group.

In the IT group, there was a significantly higher number of patients with no need for medical treatment compared to the non-IT group (70\% vs 39\%) ( $p$-value $<0.0001)$.

\section{Discussion}

A meta-analysis performed by Cingi et al. in 2014 suggests that IT is associated with a much higher reduction of symptoms compared to placebo ${ }^{(14)}$. At present, we lack information on the comparison between the degree of symptom control of AR by pharmacotherapy and IT on the long term. Anotherin a real life setting. A meta-analysis performed by Matricardi et al. in 2011 provided indirect but consistent evidence that SCIT is at least as potent as pharmacotherapy in controlling the symptoms of seasonal AR as early as the first season of treatment ${ }^{(11)}$. We here make a first attempt to evaluate the degree of symptom control, the total and individual nasal symptom severity and the current medication use in patients at 3 years after either starting medical treatment or immunotherapy. IT was associated with a higher percentage of patients with controlled AR, with mild disease and with lower medication use than those AR patients in which IT was started or ongoing. 
The strength of this study lies inrelates to the real-life character of AR treatment in a tertiary referral center, as well as in the large number of patients studied in this monocenter trial. The authors acknowledge that non- responder bias may be a problem in survey studies. We tried to minimalize the non-responder bias by calling all the 54 patients who after two months didn't reply on the questionnaire initially. There were 28 of the non-responders (52\%) who fulfilled the questionnaire after the telephone call. Seven of them $(25 \%)$ had a VAS $\geq 5$. Overall mean VAS score of the initial non-responders was 3.1. There were only few (only 9 out of 120 patients) who refused to reply because of dissatisfaction about the treatment.

Of note, the questionnaire was filled out during the Fall season, i.e. the season with increased exposure to house dust mite (HDM) exposure. Despite the higher sensitization to HDM within the IT group, the latter group showed lower scores for TNS and lower medication use. A weakness of our study includes the observational nature without a prospective evaluation of symptom controlcontrole. The patients included in this study have not been matched at baseline for severity, duration or medication use. However, the study was conducted in a tertiary referral center, and the most severe AR patients had been proposed to start IT, according to international guidelines. By not differentiating the specific allergens, this could lead to altered results and conclusions. But, we used a mixture of antigens depending on the allergen sensitization of the patients, since it was an experimental survey with not yet standardized protocols for use of immunotherapy, which implies that we could not differentiate for specific allergens. Nevertheless, the overall success rate is significantly higher in terms of severity and duration of symptoms of AR.

Based on the proposed cut-off value of VAS $<5 / 10$ for TNS (6), rhinitis was considered as being controlled in $84 \%$ of IT patients vs $63 \%$ of non-IT patients. Amongst the uncontrolled patients in the IT group (13/82), 2 suffered from concomitant asthma. For the TNS and the individual nasal symptoms we used a VAS score as suggested by Bousquet ${ }^{(9)}$, we conducted our study in 2013, but a more recent study shows that objective measures of nasal obstruction, especially Peak Nasal Inspiratory Flow, can give useful information on aspects of the disease, different from those obtained from the patient's perception, so this needs to be taken in consideration in examining the AR patients ${ }^{(16)}$. At 3 years after starting the treatment, $78 \%$ of the IT group were considered to have a mild form of AR compared to $32 \%$ in the non-IT group, with $82 \%$ of the IT group having intermittent AR vs $49 \%$ in the non-IT group. This observation is the clinical result of the allergen tolerance induced by IT and concomitant reduced symptom severity and reduced need for medication. Comparison of medication use at 3 years after starting IT or medical therapy showed that $89 \%$ of the patients who have finished the IT and $54 \%$ of the patients who were still treated with IT, did not use additional medication for AR. Strikingly, 31\% of the patients treated with immunotherapy did not use any medication for treatment of AR anymore. Therefore, more frequent consultations with increased adherence to medical treatment cannot explain the results in this study, as the majority of AR do not need medical treatment anymore at 3 years after starting IT. Our real-life observational study shows that the majority of patients with IT for AR perform better at 3 years after the onset of IT than the non-IT patients, on the level of symptom control, severity of disease and medication use. These observations are of clinical value when discussing the benefit of IT on the longterm with AR patients.

\section{Acknowledgements}

Peter W. Hellings is a fundamental clinical researcher of the FWO Vlaanderen.

\section{Authorship contribution}

VD, MT, WF and PH designed the study and conducted the study. VD, MT, SS, WF and PH analyzed the data and wrote the manuscript. All patients diagnosed and treated by $\mathrm{PH}, \mathrm{JC}$ were included.

\section{Conflict of interest}

All authors state they have no conflict of interest in relation to this study and the results described in the manuscript.

\section{References}

1. Greiner AN, Hellings PW, Rotiroti G, Scadding GK. Allergic rhinitis. Lancet 2011; 378: 2112-22.

2. Bousquet J, Khaltaev N, Cruz AA, Denburg J, Fokkens WJ, Togias A, et al. Review article Allergic Rhinitis and its Impact on Asthma ( ARIA ) 2008. Allergy 2008; 63: 8-160.

3. Calderón M a., Casale T, Cox L, Akdis C a., W. Burks a., Nelson HS, et al. Allergen immunotherapy: a new semantic framework from the European Academy of Allergy and Immunology/PRACTALL Consensus Report. Allergy. 2013; 68: 825-8

4. Wilson DR, Lima MT, Durham SR. Sublingual immunotherapy for allergic rhinitis: systematic review and meta-analysis. Allergy 2005 60: 4-12.

5. Hellings PW, Dobbels F, Denhaerynck K, Piessens M, Ceuppens JL, De Geest S. Explorative study on patient's perceived knowledge level, expectations, preferences and fear of side effects for treatment for allergic rhinitis. Clin Transl Allergy. 2012; 2: 9.

6. Bousquet PJ, Combescure C, Neukirch F, Klossek JM, Méchin H, Daures J-P, et al. Visual analog scales can assess the severity of rhinitis graded according to ARIA guidelines. Allergy 2007; 62: 367-72

7. Demoly P, Calderon M a, Casale T, Scadding
G, Annesi-Maesano I, Braun J-J, et al. Assessment of disease control in allergic rhinitis. Clin Transl Allergy. 2013; 3: 7.

8. Scadding G, Hellings P, Alobid I, Bachert C, Fokkens W, van Wijk RG, et al. Diagnostic tools in Rhinology EAACl position paper. Clin Transl Allergy. 2011; 1: 2.

9. Brozek JL, Bousquet J, Baena-Cagnani CE, Bonini S, Canonica GW, Casale TB, et al. Allergic Rhinitis and its Impact on Asthma (ARIA) guidelines: 2010 revision. J. Allergy Clin. Immunol. 2010; 126: 466-76.

10. Hellings PW, Fokkens WJ, Akdis C, Bachert C, Cingi C, Dietz de Loos D, et al. Uncontrolled allergic rhinitis and chronic rhinosinusitis: 
where do we stand today? Allergy 2013; 68: 1-7.

11. Matricardi PM, Kuna P, Panetta V, Wahn U Narkus A. Subcutaneous immunotherapy and pharmacotherapy in seasona allergic rhinitis: a comparison based on meta-analyses. J. Allergy Clin. Immunol. 2011;128(4):791-799.

Peter W. Hellings

Department of Otorhinolaryngology

Head and Neck Surgery

University Hospitals KU Leuven

Kapucijnevoer 33, 3000 Leuven,

Belgium

Tel: $+32-16-332342$

Fax: +32-16-346035

E-mail: Peter.Hellings@uzleuven.be
ADVERTISEMENT
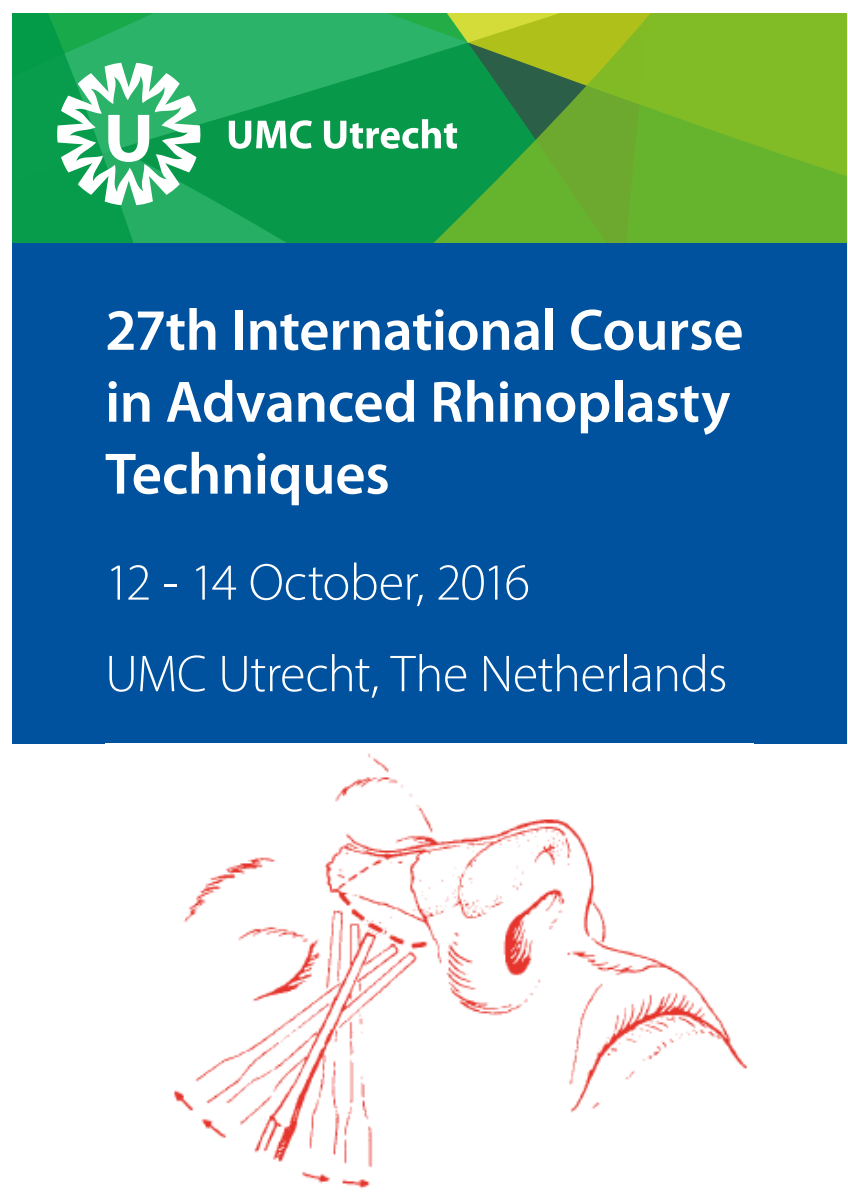

\section{Course Directors:}
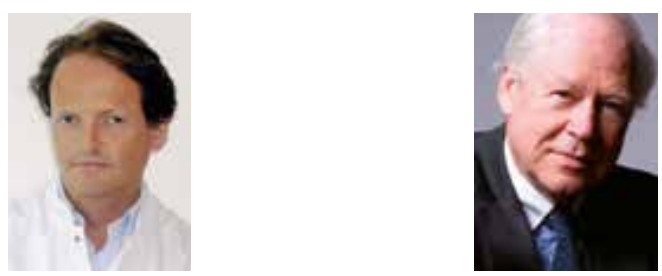

Dirk Jan Menger

Teacher of honour: David Kim, San Francisco, USA,

- multidisciplinary international faculty

- didactic lectures and expert panels

- live surgery

- fresh frozen cadaver dissection of the Nose

NEW . Workshop Chin augmentation

Preliminary facultry:

- F. Apaydin - N. Janssen $\quad$ M.J. Middelweerd

-R.L.A.W. Bleys $\quad$ D. Kim $\quad$ M. Muradin

-M. Bönisch $\quad$ M. Kon $\quad$-G.J. Nolst Trenité

-Tj.D. Bruintjes - I. Ligtenberg I.W.A. Otten

- T.R. Bull P.J.F.M. Lohuis • A. Tasman

- H.G.Gassner M.H.J.M. Majoor H.D. Vuyk

• K.J.A.O.Ingels $\quad$ D.J.Menger $\quad$ C.C.Wever

Information: rhinoplasty@umcutrecht.nl website: www.advancedrhinoplasty.nl

箵

Universitair Medisch Centrum Utrecht 